\title{
Patty Pravo: Eccomi. 1 Day/Warner Music Italia 5054197002328, 2016.
}

Vor genau 50 Jahren machte mit dem Titel „Ragazzo triste“ eine junge Sängerin in Italien auf sich aufmerksam und wurde quasi über Nacht zu einem der Idole der Beat-Generation. Von den italienischen Medien im Laufe der Jahre mit Attributen wie „la ragazza del Piper“", „Patty la trasgressiva“ oder „l'ultima diva della canzone“ belegt, ist die musikalische Laufbahn der gebürtigen Venezianerin Nicoletta Strambelli, besser bekannt unter ihrem Künstlernamen Patty Pravo, von Höhen, aber auch einigen Tiefen geprägt. Titel wie „La bambola“, „Pazza idea“, „Pensiero stupendo“ oder „E dimmi che non vuoi morire“ wurden große kommerzielle Erfolge und auch Klassiker im Repertoire der Sängerin, deren unverwechselbares Markenzeichen die tiefe Stimme mit einem dennoch samtigen Timbre ist.

Die musikalische Produktion der Interpretin zeichnet sich durch eine kontinuierliche Weiterentwicklung aus und beinhaltet sowohl die Zusammenarbeit mit namhaften cantautori (wie beispielsweise Ivano Fossati, Franco Battiato oder Vasco Rossi) als auch immer wieder Projekte mit jungen Textern und Komponisten, die dem Repertoire Patty Pravos neue Klangfarben verleihen. Das Jahr 2016 ist für die Künstlerin nun der Anlass, ihr 50-jähriges Bühnenjubiläum zu feiern. Dieses geht einher mit einer Tournee und der Veröffentlichung eines neuen Albums mit dem Titel Eccomi. ${ }^{2}$ Mit dem darauf enthaltenen Titel „Cieli immensi“ stellte sich die Interpretin darüber hinaus dem Wettbewerb des diesjährigen Festivals von Sanremo, wo sie den 6. Platz belegte und mit dem Premio Mia Martini der Kritiker ausgezeichnet wurde.

Das von Michele Canova Iorfida produzierte neue Album versammelt 12 neue Canzoni aus der Feder verschiedener Autoren und Komponisten; hinzu kommt als Bonustrack eine Neuaufnahme von „Tutt'al più“, einem Erfolg Patty Pravos von 1970, der in einer neu arrangierten Version mit dem Rapper Fred de Palma eingesungen und im Rahmenprogramm des Festivals von Sanremo präsentiert wurde.

Das Album wird von „A parte te“ eröffnet, einer Ballade über eine Liebesbeziehung, in der sich die anfängliche Leidenschaft in Langeweile und Alltäglichkeit verwandelt hat. Unfähig, eine Entscheidung zu treffen und die Beziehung zu beenden, fragt sich die Protagonistin, wie es soweit kommen konnte und was von der Liebe noch geblieben ist. Patty Pravo interpretiert die Canzone zurückhaltend und getragen und unterstreicht dadurch den melancholischen Grundcharakter. In den Schlusstönen fällt auf, dass die Interpretin in eine höhere Tonlage wechselt, wodurch ein Hauch von Leichtigkeit in die Canzone kommt. Es folgt der Titel „Ci rivedremo poi“, der es hinsichtlich Stil und Arrangement durchaus mit den großen Produktionen der europäischen Popmusik aufnehmen kann. 
Während in den einzelnen Strophen, unterstrichen durch den durchgehenden Gebrauch des imperfetto, die Jugend der beiden Protagonisten thematisiert wird, blickt im Refrain das Ich der Protagonistin in die Zukunft, in der die beiden wieder zusammenkommen: „cammineremo insieme se vorrai“. Ergänzt werden diese beiden Perspektiven durch eine Zwischenstrophe nach der zweiten Wiederholung des Refrains, in der die Gegenwart im Zentrum steht: „ora giro a vuoto sola / dentro un vecchio tram“. Der Perspektivenwechsel wird somit durch den Zeitengebrauch - das futuro semplice im Refrain und das Präsens in der Zwischenstrophe - verdeutlicht. Auch in dieser Canzone bringt die zurückhaltende und nuancierte Interpretation Patty Pravos den intimen Charakter des Textes gut zur Geltung. Mit „Qualche cosa di diverso“ ist eine weitere Ballade vertreten, die nun aber vor allem durch ihr Arrangement besticht. Da zu Beginn das Klavier dominiert und erst im Refrain weitere Instrumente die musikalische Begleitung verstärken, rückt die Stimme der Interpretin deutlich in den Vordergrund, denn während sie in den Strophen leise und zurückhaltend eingesetzt wird, gewinnt sie im Refrain deutlich an Kraft und Nachdruck, ehe sie zum Ende hin wieder zurückgenommen wird. Durch ein noch stärker ausgefeiltes Arrangement fällt der anschließende Sanremo-Beitrag, „Cieli immensi“, auf: So wird in einigen Passagen - vor allem in den Strophen - die Stimme der Interpretin quasi verdoppelt, indem Kopf- und Bruststimme parallel aufgenommen werden $;^{3}$ der Stimme wird dadurch im Verhältnis zur musikalischen Begleitung mehr Volumen zuteil. Darüber hinaus lässt sich beobachten, dass das Arrangement auf eine gewisse Leichtigkeit angelegt ist, die sich in hervorragender Weise mit dem Begriff ,cielo', der im Text allgegenwärtig ist, verbindet. Es scheint, als flöge die Stimme der Interpretin geradezu durch die einzelnen Strophen und Refrains, was der Canzone einen ganz eigenen Charakter verleiht. Die Coda, in der das Volumen der Stimme wie auch das musikalische Arrangement deutlich zurückgenommen werden, setzt indes einen besonderen Schlusspunkt, indem sie den HörerInnen den Eindruck vermittelt, nach dem Höhenflug wieder sanft auf dem Boden zu landen. Im modernen Arrangement präsentiert sich auch das folgende „Per difenderti da me“, für das Patty Pravos Kollege Tiziano Ferro verantwortlich zeichnet. Einmal mehr geht es um eine Paarbeziehung, die jedoch letzten Endes zur Unmöglichkeit wird („Non voglio vivere così / Dimentichiamoci cosi“), und einmal mehr unterstreicht die Interpretation Patty Pravos den melancholischen Grundcharakter der Canzone, der durch das begleitende Arrangement nicht unmittelbar zutage tritt.

Mit „Nuvole“ folgt eine Canzone, in der es um die Unsicherheit der eigenen Gefühle geht und die trotz ihrer musikalischen Schlichtheit und einer zurückhaltenden Interpretation eine sehr hohe Intensität hat: Als HörerIn hat man beinahe den Eindruck, als wäre man selbst Adressat des Textes und nähme Teil an einem imaginären Dialog, was auch daran liegt, dass der Text in den Strophen zwischen Sprechen und Gesang pendelt. Lediglich im Refrain, in dem der Monolog zum Gesang wird, setzt die Interpretin ihre Stimme nachdrücklicher und kraftvoller ein und schafft so einen interessanten Kontrast zu den Strophen. Am Ende der Canzone schließt sich gewissermaßen der Kreis, indem die Ausgangssituation wieder aufgegriffen wird. Ungewöhnliche Töne schlägt im Anschluss die Canzone „Non siamo 
eroi“ an, die Patty Pravo zusammen mit dem sizilianischen Rapper Emis Killa interpretiert: Während letzterer für die typischen Rap-Klänge in den Strophen sorgt, interpretiert Patty Pravo den Refrain im ,klassischen' melodischen Stil und schafft damit einen deutlichen musikalischen Kontrast. Patty Pravo setzt damit eine Tradition fort, die ihr im Laufe ihrer fünfzigjährigen Karriere immer wieder Ausflüge in andere Musikstile beschert hat. ${ }^{4}$ Darüber hinaus könnte die Canzone auch als Angebot an die jüngere Generation verstanden werden, die sich für andere Musikrichtungen als das klassische Repertoire Patty Pravos begeistert. Der darauffolgende Titel „Possiedimi“ ist ebenfalls eine Art musikalischer Brückenschlag, stammen doch Text und Melodie von der italienischen Rocksängerin Gianna Nannini. Die Canzone selbst ist jedoch keineswegs rockiger Natur, sondern eher als moderne Popballade arrangiert. Im Text fallen die zahlreichen Imperative auf, die mit einer inhaltlichen Überraschung einhergehen, kann doch die Botschaft der Canzone als freiwillige Unterordnung gegenüber dem Partner verstanden werden: „Non darmi nessuna libertà / Ora che ho imparato finalmente a dire amore mio“. Die Stimme der Interpretin wirkt jedoch keineswegs aggressiv oder fordernd, sondern unterstreicht eher die Abhängigkeit, die im Text allgegenwärtig scheint. Einen deutlichen Kontrast dazu bildet im Anschluss die Canzone „Se chiudo gli occhi“. Es handelt sich um eine weitere Ballade, in der das musikalische Arrangement in den Strophen zugunsten der Stimme der Interpretin in den Hintergrund tritt. Die Canzone handelt von der Schwierigkeit, Gefühle adäquat zu beschreiben: „Come è difficile disegnare un'emozione sopra un foglio / Così una canzone non potrà descrivere l'amore“. Gerade die Canzone selbst ist es aber am Ende des Textes, die zum gemeinsamen Band zwischen zwei Liebenden wird, deren Liebe keine Dauerhaftigkeit beschert ist. „Come una preghiera“ präsentiert sich wiederum im Stil moderner Popmusik und hebt sich von der vorhergehenden Ballade durch seinen deutlich rockigen Charakter ab. Dem Arrangement entsprechend ist bei diesem Titel auch die Stimme der Interpretin deutlich kraftvoller und energischer, ohne dabei aber ihr typisches Timbre einzubüßen: der ,Pravo-Stil' ist auch hier beim ersten Hören zu erkennen. Mit „Se“ schließt sich noch einmal eine Ballade an, die unter dem Motto „Se nascessi un'altra volta“ steht und davon handelt, dass es nichts zu bereuen gibt und man in einem neuen Leben wieder genauso handeln würde. Die Stimme der Interpretin ist zwar etwas getragener als in der vorhergehenden Canzone, ohne aber deshalb in übertriebenes Pathos zu verfallen. „Un uomo semplice“, der letzte reguläre Titel des Albums, kommt im Kontrast dazu beinahe heiter daher, was zum Großteil der Melodie und dem Arrangement geschuldet ist, da der Text durchaus eine ernste Dimension hat. Doch auch die Interpretation Patty Pravos passt sich der Leichtigkeit des Arrangements an, wodurch ähnlich wie bei „Cieli immensi“ die Stimme der Sängerin etwas Schwebendes bekommt.

Der bereits erwähnte Bonus-Track „Tutt’al più “ ist das Produkt einer Zusammenarbeit mit einem weiteren Vertreter der italienischen Rap-Szene, Fred de Palma. Dieser hat die Canzone um neue Strophen im Rap-Stil ergänzt, die mit dem Refrain aus dem Original kombiniert werden (letzterer wird, wie schon bei „Non siamo eroi“, von Patty Pravo interpretiert bzw. stimmlich dominiert). Auch in diesem Fall wird eine Brücke zwischen ,neuer und ,alter' Canzone geschlagen, was ein durchaus interessantes Experiment darstellt. 
Mit Eccomi ist Patty Pravo ein überzeugendes Album gelungen, für das sich das Warten nicht nur für überzeugte Fans - gelohnt hat. Es zeigt sich einerseits, dass die ,alte' Generation der italienischen Canzone durchaus mit dem musikalischen Nachwuchs mithalten kann, zum anderen aber auch, dass sich die Sängerin kontinuierlich weiterentwickelt und ihrem Repertoire immer wieder neue Facetten hinzufügt, ohne dabei ihre Funktion als Interpretin bzw. Vermittlungsinstanz zu vernachlässigen.

\section{Andreas BONNERMEIER (Mainz)}

\section{Endnoten}

1 Der „Piper Club“ war vor allem in den 1960er Jahren ein angesagtes Musiklokal in Rom, in dem regelmäßig viele Größen der Musikszene auftraten, das aber auch dem musikalischen Nachwuchs eine Plattform bot.

2 Das vorletzte Album der Künstlerin mit dem Titel Nella terra dei pinguini war im Jahr 2011 erschienen.

3 In der Vergangenheit wurde dieses Verfahren u.a. in einigen Canzoni der Sängerin Alice eingesetzt, beispielsweise in „Prospettiva Nevski“ und „Summer on a solitary beach“ (beide aus dem Jahr 1984) oder „Una notte speciale“ (1981).

4 Beispiele hierfür sind das 1979 erschiene Munich Album, das von den Deutschen Michael Holm und Rainer Pietsch im typischen Disco-Sound der späten 1970er Jahre produziert wurde, oder auch das Album Ideogrammi aus dem Jahr 1994, das in China entstand. 